\title{
What is the Right Illumination Normalization for Face Recognition?
}

\author{
Aishat Mahmoud Dan-ali \\ Department of Computer Science and Engineering \\ The American University in Cairo \\ AUC Avenue, P.O. Box 74, New Cairo 11835, Egypt
}

\author{
Mohamed Moustafa \\ Department of Computer Science and Engineering \\ The American University in Cairo \\ AUC Avenue, P.O. Box 74, New Cairo 11835, Egypt
}

\begin{abstract}
In this paper, we investigate the effect of some illumination normalization techniques on a simple linear subspace face recognition model using two distance metrics on three challenging, yet interesting databases. The research takes the form of experimentation and analysis in which five illumination normalization techniques were compared and analyzed using two different distance metrics. The performances and execution times of the various techniques were recorded and measured for accuracy and efficiency. The illumination normalization techniques were Gamma Intensity Correction (GIC), discrete Cosine Transform (DCT), Histogram Remapping using Normal distribution (HRN), Histogram Remapping using Log-normal distribution (HRL), and Anisotropic Smoothing technique (AS). Results showed that improved recognition rate was obtained when the right preprocessing method is applied to the appropriate database using the right classifier.
\end{abstract}

Keywords-face recognition; preprocessing; illumination

\section{INTRODUCTION}

Illumination and pose challenges have been the serious bottlenecks in face recognition algorithms. Many attempts were made to overcome the effects of these challenges; however, the perfect face recognition system invariant to all challenges is still elusive. What researchers concentrate on is trying to overcome a couple of challenges at a time.

This paper is mainly focused on studying how a face recognition system is being affected by illumination variation. Attention is concentrated on the preprocessing part of the system. Different image preprocessing techniques for face recognition were proposed and experimented with. The sequence of execution of the proposed method includes the preprocessing step, PCA/LDA subspace [12], and cosine/Euclidean classifiers.

To facilitate a comprehensive study and analysis, five different preprocessing techniques were implemented on the PCA/LDA model using two different classifiers. These preprocessing techniques were carefully chosen based on their popularity and recorded success.

This yielded ten (10) set of experimentations on each of the three databases used. The preprocessing techniques are Gamma Intensity Correction (GIC), Discrete Cosine Transform (DCT), Histogram remapping with normal distribution (HRN), Histogram remapping with log-normal distribution (HRL) and Anisotropic smoothing technique (AS).

\section{Preprocessing Methods For Face ReCOGNition}

Preprocessing plays a vital role in face recognition systems, because it always tries to bring the test images and those in the database into a normalized canonical form. The use of preprocessing in face recognition is generally used to overcome the effect of lighting, enhancing image contrast and normalizing the image in terms of rotation and scale. Fig. 1 shows an example of how different illumination conditions of the same person make face recognition a difficult task, even for humans.

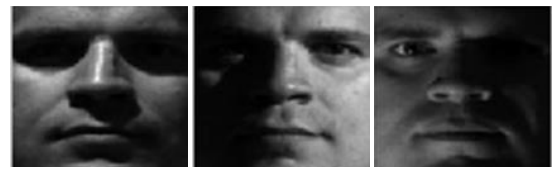

Fig. 1. Example of images from the Yale B database with different illumination conditions

We have seen recently a comparison of some preprocessing steps in combination with some face matching methods reported in [13]. In this paper, we are focusing on other set of preprocessing methods combined with a simple matcher based on cosine or Euclidean distances.

The preprocessing methods used in this work are highlighted below

\section{A. Gamma Intensity Correction (GIC)}

Gamma intensity correction is used to control the overall brightness of an image by changing the gamma parameter and it can be used to correct the lighting variations in the face image [7]. The gamma correction is the process of taking the exponential of the input image. The output image would be darker or brighter depending on the value of gamma $\gamma$. In this work a value of gamma $=0.2$ has been used. Gamma correction has been used in [2] and [7] for illumination normalization.

\section{B. Discrete Cosine Transform (DCT)}

The Discrete Cosine Transform is a novel approach for illumination normalization under varying lighting conditions used in face recognition algorithms that keeps facial features intact while removing excess lighting variations [1], [6]. It is a popular technique for image compression in which low frequency DCT coefficients that are correlated with illumination variations are curtailed thereby significantly 
reducing the effect of illumination variation. Example of application of DCT is in JPEG image compression.

\section{Histogram Remapping techniques}

This is a variation of the histogram equalization (HE) method in which other distributions are used instead of the default uniform distribution that is being used in the HE method. These distributions include normal, log-normal and exponential distribution. To investigate the possibilities of these distributions experiments were conducted using the Normal distribution and Log-normal distribution in the histogram remapping algorithm as suggested by this paper [9].

\section{Anisotropic Smoothing}

This technique is based on the reflection perception model. This work was pioneered by Gross and Brajovic [3] in which they found an estimate of the luminance $\mathrm{L}(\mathrm{x}, \mathrm{y})$ such that reflectance $\mathrm{R}(\mathrm{x}, \mathrm{y})$ is produced by dividing the input image $\mathrm{I}(\mathrm{x}$, y) by $\mathrm{L}(\mathrm{x}, \mathrm{y})$. These ensure that the local contrast is suitably improved. They obtained this by enforcing a smoothing constraint on the algorithm.

\section{EXPERIMENTS}

In this section, experiments were carried out that illustrate the effectiveness of the proposed method using three publicly available face databases with considerable illumination variations, the databases are: CAS PEAL database [8]; Extended Yale Face Database B ('Extended Yale-B') [10]; AT\&T Database [4]. The sequence of execution includes preprocessing in conjunction with the linear subspace model, and classification using either of the classifiers. The standard protocol used in evaluating the result is also given.

\section{A. Experimental Setup}

In these experiments only frontal face views were used in the experiment, but lighting, expression and identity may all vary. All of the images in the CAS PEAL-R1 lighting subset, and all the images were geometrically normalized before preprocessing. While for the AT\&T database, the images were 8 bit gray-scale images containing hair and ear regions. All the images in the databases were resized to $100 \mathrm{x} 100$ pixels.

For the testing, analysis and evaluation of the proposed method, a toolbox containing MATLAB scripts named "The $\mathrm{PhD}$ face recognition toolbox" was partially used. The tool was made publicly available free of charge by V. Struct [5]. Another collection of MATLAB face recognition files called "FaceRecEvaluator" was also utilized. The FaceRecEvaluator was developed by Brian and Enrique [11], and is available freely for use in academic and research domains.

\section{B. Results}

Here, analysis and discussion of the various performances i.e. recognition accuracy and execution time of the techniques employed are highlighted. Five (5) preprocessing techniques and two (2) distance metrics were experimented with using these databases; the preprocessing techniques are Gamma intensity correction (GIC), Discrete Cosine Transform (DCT), Histogram remapping using Normal distribution (HRN), Histogram remapping using Log-normal distribution (HRL), and Anisotropic Smoothing technique (AS). The distance metrics are Euclidean (EUC) and cosine (COS) distance metrics. In total we have, ten (10) set of experiments using these databases namely: (GIC+EUC), (GIC+COS), (DCT+EUC), (DCT+COS), (HRN+EUC), (HRN+COS), (HRL+EUC), (HRL+COS), (AS+EUC), (AS+COS).

The analysis and discussion is structured according to each of the three databases CAS PEAL, Extended Yale B, and ATT used and the five illumination normalization techniques with the two different distance metrics. This produces 60 different set of experiments.

\section{CAS PEAL Database}

The CAS PEAL contains six (6) different subsets in the frontal category. Ten (10) images of 77 subjects making a total of 770 images were used from the Lighting subset. Ten (10) experiments were conducted using this database and the result is as follows:

As shown in Fig. 2, Gamma Intensity correction together with Euclidean distance yielded the second best performance with $64.9 \%$ recognition rate in $3.2 \mathrm{~ms} / \mathrm{img}$, while Gamma Intensity correction together with Cosine distance gave $62.7 \%$ recognition rate in $16.5 \mathrm{~ms} / \mathrm{img}$. However, Discrete Cosine Transform in either case i.e., with Euclidean or Cosine distance metric gave the lowest performance for this database. DCT with Euclidean distance produces $10.4 \%$, while DCT with Cosine distance produces $17.2 \%$ in $3.1 \mathrm{~ms} / \mathrm{img}$ and $15.1 \mathrm{~ms} / \mathrm{img}$ respectively. Histogram remapping using normal distribution with Euclidean distance gave 63\% recognition accuracy in 3.3 $\mathrm{ms} / \mathrm{img}$ while Histogram remapping using normal distribution with cosine distance yielded the best performance for this database with $65.6 \%$ recognition rate in $15.8 \mathrm{~ms} / \mathrm{img}$. However, the only minor setback of this composition (HRN + Cos) is the execution time, which is slower than the (HRN + Euc) combination.

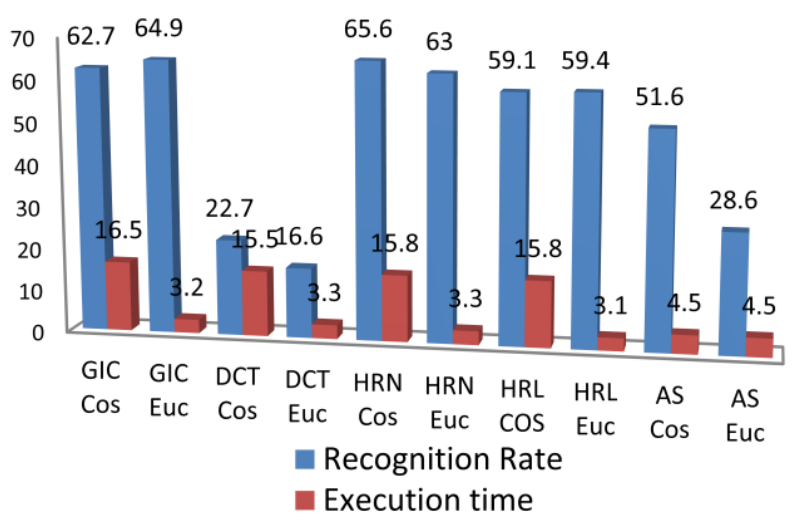

Fig. 2. Performances of different preprocessing techniques on the CAS PEAL database using two distance metrics

On the other hand, histogram remapping using Log-normal distribution with cosine distance generates $59.1 \%$ recognition accuracy in $15.3 \mathrm{~ms} / \mathrm{img}$, while this method together with the Euclidean distance produces 59.4 in $3.1 \mathrm{~ms} / \mathrm{img}$. Is it important to note that using this technique the Euclidean distance gives better performance in terms of accuracy and execution time. 
The last experiment conducted with this database is the Anisotropic Smoothing (AS) method. This technique was also applied using the Euclidean and Cosine distance metrics. The first experiment with Euclidean distance measure yielded an accuracy of $51.6 \%$ in $4.5 \mathrm{~ms} / \mathrm{img}$, while the second experiment produced $28.6 \%$ in $4.5 \mathrm{~ms} / \mathrm{img}$ with the cosine distance measure.

\section{Extended Yale B database}

As noted earlier the Extended Yale database is divided into subsets as initially suggested by the authors of the database. These experiments were conducted on subset 2 to subset 5 of the database and the results were analyzed separately.

1) Yale $B$ subset 2 result: This subset is not very challenging, therefore; all of the techniques except DCT yielded $100 \%$ recognition accuracy with varying execution times, while DCT produce $96.7 \%$ in both cases.

2) Yale B subset 3 result: This subset contains images that have more illumination variation than the previous subset. The GIC plus Euclidean and the GIC plus the cosine distance generates $100 \%$ recognition rates each in $1 \mathrm{~ms} / \mathrm{img}$ and $2.1 \mathrm{~ms} / \mathrm{img}$ respectively. A recognition rate of $83.6 \%$ in $2 \mathrm{~ms} / \mathrm{img}$ was obtained from the DCT method using the cosine distance, while $78.9 \%$ was obtained from this subset using $\mathrm{DCT}+$ Euc combination in 1ms/img. In the histogram remapping technique using normal distribution, a recognition rate of $100 \%$ was also obtained in both cases using the two distance metrics. The execution times differ with $0.9 \mathrm{~ms} / \mathrm{img}$, with Euclidean distance having $1.1 \mathrm{~ms} / \mathrm{img}$ and cosine distance having 2ms/img. Histogram remapping using log-normal distribution yielded $100 \%$ recognition rate in $1.9 \mathrm{~ms} / \mathrm{img}$ using cosine distance and same method using Euclidean distance gave same result but in $1 \mathrm{~ms} / \mathrm{img}$. The Anisotropic smoothing technique is the final experiment carried-out using this subset. The result obtained showed a recognition rate of $99.3 \%$ in $1.5 \mathrm{~ms} / \mathrm{img}$ and $98 \%$ in $1.1 \mathrm{~ms} / \mathrm{img}$ using the cosine and Euclidean distance metrics.

3) Yale $B$ subset 4 result: The fourth subset of this database contains images with second highest degree of illumination variation. As shown in Fig. 3, GIC + Cos yielded a recognition rate of $92.1 \%$ in $2.1 \mathrm{~ms} / \mathrm{img}$, while GIC + Euc $94.7 \%$ in $1.1 \mathrm{~ms} / \mathrm{img}$. It can be realized here also that the Euclidean distance gave the best performance. In DCT experiments, DCT + Cos produces $64.5 \%$ in $2 \mathrm{~ms} / \mathrm{img}$ while, DCT + Euc generates $51.3 \%$ in $0.9 \mathrm{~ms} / \mathrm{img}$. Analyzing this and the previous result under this technique closely, one can see that the DCT method is best matched with the cosine distance measure. On the other hand, experiments with histogram remapping using the normal distribution (HRN + Euc) on this subset produce $80.3 \%$ in $0.9 \mathrm{~ms} / \mathrm{img}$ using Euclidean distance and $(\mathrm{HRN}+\mathrm{Cos})$ produces $78.3 \%$ in 2 ms/img using cosine distance. Similar results were obtained using this remapping technique with lognormal distribution. Experiments with cosine distance produce $78.3 \%$ in 2 ms/img, while that of Euclidean distance produce $78.9 \%$ in 1 ms/img. Between these two methods the HRN + Euc gives the best result. The last experiment on this subset is the anisotropic smoothing method. The AS + cos technique gives $82.9 \%$ in $1.5 \mathrm{~ms} / \mathrm{img}$ while the AS + Euc gives $86.5 \%$ in 1.3 $\mathrm{ms} / \mathrm{img}$. It can be noted that the anisotropic technique (AS) perform better than the three methods on this subset; that is the DCT, HRN and HLN. However the gamma intensity correction (GIC) out perform all the techniques on this subset.

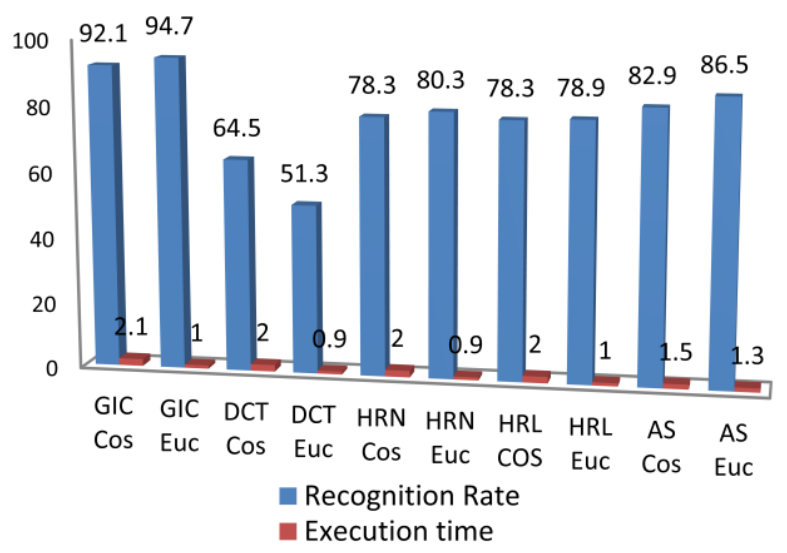

Fig. 3. Performances of different preprocessing techniques on subset 4 of the Yale B database using two distance metrics

4) Yale B subset 5 result: This subset is the last and most complex of the extended Yale B database. As depicted in Fig. 4, GIC with cosine distance produces $95.4 \%$ recognition rate in $2 \mathrm{~ms} / \mathrm{img}$ whereas GIC with Euclidean distance produces the same result in $1 \mathrm{~ms} / \mathrm{img}$. The next experiment is the DCT with the cosine distance measure which gave a recognition accuracy of $59.2 \%$ in $2 \mathrm{~ms} / \mathrm{img}$ while the DCT + euc drops performance by generating only $50 \%$ recognition rate in $1 \mathrm{~ms} / \mathrm{img}$. It is relevant to point out that the DCT always perform better when combined with cosine distance metric. In the next set of experiments histogram remapping with normal distribution (HRN) was applied to this subset, the result indicated HRN perform better than HRL in both cases using the two distance metrics. HRN+euc gave $80.3 \%$ in $1 \mathrm{~ms} / \mathrm{img}$ whereas $\mathrm{HRN}+\cos$ gave $77.6 \%$ in $2 \mathrm{~ms} / \mathrm{img}$. Similarly, HRL with cosine distance produced $77.6 \%$ in $2 \mathrm{~ms} / \mathrm{img}$ and HRL+euc produced $73.7 \%$ in $0.9 \mathrm{~ms} / \mathrm{img}$. In the anisotropic smoothing experiment, the AS + cos combination yields an amazing $92.1 \%$ recognition accuracy in $1 \mathrm{~ms} / \mathrm{img}$, and the AS + euc method yields $90.8 \%$ recognition accuracy in just $1 \mathrm{~ms} / \mathrm{img}$. It can be gathered from the experiments conducted on this complex subset that Gamma Intensity correction performed better that all the techniques used on the subset..

\section{E. AT\&T database}

This database was used as a means of assessing the performance of the techniques on a database devoid of any illumination challenge. However, the database contains slight variations due to pose, accessories (glasses), and expression. As shown in Fig. 5, the gamma Intensity correction with the cosine distance yielded $95.6 \%$ in $1.4 \mathrm{~ms} / \mathrm{img}$, while the same technique with Euclidean distance yielded $94.4 \%$ in 0.9 
ms/img. DCT technique with the cosine distance resulted in $28.8 \%$ recognition rate in $1.5 \mathrm{~ms} / \mathrm{img}$ whereas DCT +euc indicated a recognition accuracy of $28.8 \%$ in $0.7 \mathrm{~ms} / \mathrm{img}$. In histogram remapping techniques the following results were obtained: $\mathrm{HRN}+\cos$ produced $95 \%$ in $1.5 \mathrm{~ms} / \mathrm{img}$ recognition accuracy whereas HRN + euc produced $95 \%$ accuracy in $0.8 \mathrm{~ms} / \mathrm{mg}$. In the same way, HRL $+\cos$ resulted in $95 \%$ recognition rate in $1.4 \mathrm{~ms} / \mathrm{img}$ while HRL+euc resulted in $95 \%$ accuracy in $0.7 \mathrm{~ms} / \mathrm{img}$. These show that the remapping techniques produce somewhat similar results with little difference in execution time. Anisotropic smoothing technique result indicated a drop in recognition rate from the previous results; AS+cos produced $87.5 \%$ in $1.5 \mathrm{~ms} / \mathrm{img}$ but $\mathrm{AS}+$ euc produced $86.3 \%$ in $0.7 \mathrm{~ms} / \mathrm{img}$.

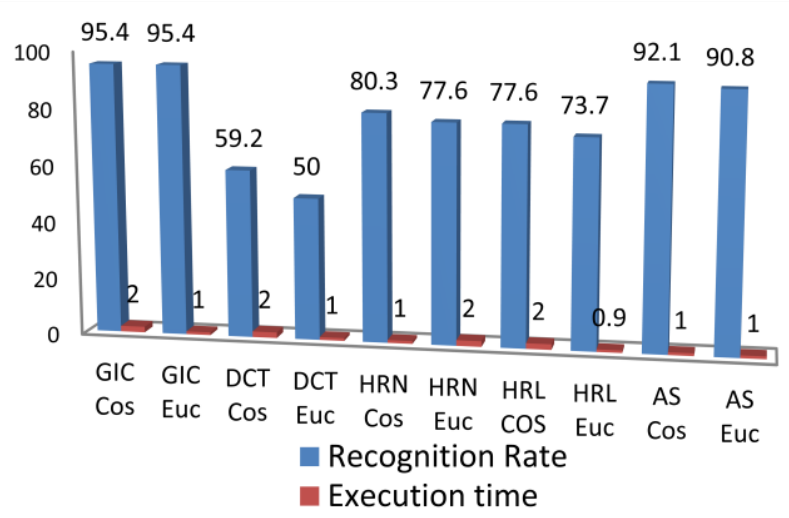

Fig. 4. Performances of different preprocessing techniques on subset 5 of the Yale B database using two distance metrics

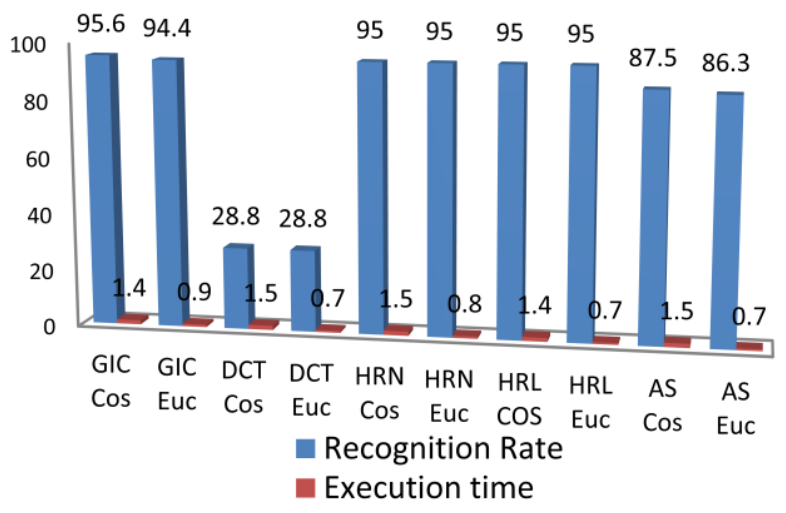

Fig. 5. Performances of different preprocessing techniques on the AT\&T database using two distance metrics

\section{CONCLUSIONS}

In this paper, different preprocessing techniques for face recognition systems have been proposed and implemented using hybrid approach and linear subspace modeling for feature extraction, and dimensionality reduction and cosine or Euclidean distance metric for classification. The proposed preprocessing techniques are Histogram Remapping using Normal distribution (HRN) and Histogram Remapping using Log-normal distribution (HRL). Other preprocessing techniques experimented with are Gamma Intensity Correction
(GIC), Discrete Cosine transform (DCT), and Anisotropic Smoothing (AS). The above mentioned techniques resulted in ten types of face recognition methods: (GIC+EUC), $(\mathrm{GIC}+\mathrm{COS}), \quad(\mathrm{DCT}+\mathrm{EUC}), \quad(\mathrm{DCT}+\mathrm{COS}), \quad(\mathrm{HRN}+\mathrm{EUC})$, (HRN+COS), (HRL+EUC), (HRL+COS), (AS+EUC), $(\mathrm{AS}+\mathrm{COS})$.

The performances of these ten methods have been evaluated in terms of percentage of recognition accuracy, and for the total execution time to monitor efficiency. The following conclusions are made based on the results and analyses of the above mentioned face recognition techniques:

- The Gamma Intensity correction provides good performance on all the databases particularly those with extreme illumination condition like the subset 4 and subset 5 of the Extended Yale B database. However, the technique was outperformed by histogram remapping using normal distribution on the CASPEAL lighting subset. Generally, the GIC method performed at its best when combined with the Euclidean distance metric, i.e. the (GIC + EUC) arrangement.

- The Histogram remapping technique play a vital role in the CASPEAL complex database in which the lighting and illumination variation is at the extreme with some images very much over-exposed while others are very much under-exposed. In this category of illumination variation technique, the HRN performed better than the HRL in almost all the cases. HRN provides better results when combined with the cosine distance metric.

- The Discrete cosine transform method (DCT) generates the worst recognition accuracy in all the databases used. It can be concluded from these set of experiments that this method is not the best in terms of illumination normalization for face recognition purposes. However, despite this low performance, the DCT method perform better when merged with the cosine distance metric even though it takes some time to finish execution.

- The Anisotropic Smoothing technique (AS) provides second best performance on the Yale B database subsets, however, not so good result was obtained when applied to the CASPEAL lighting subset. This method work well when combined with the Cosine distance metric.

- Between the two distances metrics studied, the cosine distance produce superior performance in almost all the experiments carried out with some minor exceptions like in the GIC technique. The only drawback of this method is the execution time with is slower than the Euclidean distance measure.

\section{REFERENCES}

[1] W. Chen, Er. M. J, and S. Wu, "Illumination compensation and normalization for robust face recognition using discrete cosine transform in logarithm domain," IEEE Transactions on Systems, Man and Cybernetics, vol.36, no.2, 2006, pp. 458-466.

[2] X. Tan, and B. Triggs, "Enhanced local texture feature sets for face recognition under difficult lighting conditions," In Proceedings of the IEEE international workshop on analysis and modeling of faces and gestures, 2007. pp. 168-182. 
[3] R. Gross, V. Brajovic, "An image preprocessing algorithm for illumination invariant face recognition". In: Kittler, J., Nixon, M.S. (eds.) AVBPA 2003 LNCS, vol. 2688, 2003.pp. 10-18. Springer, Heidelberg

[4] S. Ferdinando, H. Andy, "Parameterisation of a Stochastic Model for Human Face Identification." Proceedings of 2nd IEEE Workshop on Applications of Computer Vision, Sarasota FL, December 1994.

[5] V. Štruc, N. Pavešic, "Photometric normalization techniques for illumination invariance", in: Y.J. Zhang (Ed), Advances in Face Image Analysis: Techniques and Technologies, IGI Global, 2011. pp. 279-300.

[6] Ruiz-del-Solar, J., Quinteros, J., Comparing Pre-Processing Approaches for Illumination Invariant Face Recognition, Technical Report UCHDIE-VISION-2006-04, Dept. Elect. Eng., Universidad de Chile. Available (August 2006) in: http://vision.die.uchile.cl/

[7] S. Shan, W., Gao, B., Cao, D., Zhao, "Illumination normalization for robust face recognition against varying lighting conditions", In: Proc. IEEE Workshop on AMFG, 2003, pp157-164.

[8] W. Gao, S. Member, B. Cao, S. Shan, X. Chen, D. Zhou, X. Zhang, and D. Zhao, "The CAS-PEAL Large-Scale Chinese Face Database and
Baseline Evaluations," vol. 38, no. 1, 2008, pp. 149-161.

[9] V. Štruc, J. Žibert, and N. Pavešic, "Histogram remapping as a preprocessing step for robust face recognition", WSEAS transactions on information science and applications, vol. 6, no. 3, pp. 520-529, 2009."

[10] A. S. Georghiades, and P.N. Belhumeur, and D.J. Kriegman, "From Few to Many: Illumination Cone Models for Face Recognition under Variable Lighting and Pose", "IEEE Trans. Pattern Anal. Mach. Intelligence", volume 23, number 6, pages "643-660",2001

[11] B.C., Becker, E.G Ortiz. "Evaluation of Face Recognition Techniques for Application to Facebook," in Proceedings of the 8th IEEE International Automatic Face and Gesture Recognition Conference, 2008.

[12] P.N Belhumeur, J.P Hespanha, and D.J Kriegman, "Eigenfaces vs. Fisherfaces: recognition using class specific linear projection," IEEE Transactions on pattern analysis and machine, vol.19, 1997, pp.711720.

[13] Hu Han, Shiguang Shan, Xilin Chen, Wen Gao, "A comparative study on illumination preprocessing in face recognition", Pattern Recognition, vol. 46, no. 6, 2013, pp. 1691-1699. 